\title{
Medroxiprogesterona en el manejo de S.O.A.
}

\author{
Dres.: Gustavo Gómez T.* Enrique Herrera C. ** y Raúl Escobar N.**
}

El tratamiento en el Síndrome de Ovario Androgénico (S.O.A.) está dirigido principalmente a tres objetivos: regularizar los ciclos, tratar la infertilidad secundaria a la anovulación y corregir el hirsutismo. En un trabajo anterior (1) informamos el resultado del tratamiento con diferentes drogas e hicimos alguna observación inicial cuando se trataban pacientes que no querían embarazarse sino que su única finalidad era regularizar sus ciclos. Ahora analizamos los resultados de un grupo de pacientes tratadas con medroxiprogesterona en los cuales el objetivo principal era regularización de los ciclos.

\section{PACIENTES Y METODOS}

Se realizó en 30 pacientes de la Clínica de Endocrinología Ginecológica e Infertilidad en las que se llegó al diagnósti-

Profesor Asociado Departamento de Obstetricia y Ginecología. Coordinador Clí. nica Endocrinología e Infertilidad. Hospital Universitario del Valle. Cali, Colombia.

* Residente Tercer Año Departamento de Obstetricia y Ginecología. Hospital Universitario del Valle. Cali, Colombia. co de Sindrome de Ovario Androgénico. Los parámetros para este diagnóstico fueron:

a) Historia Clínica de oligo-amenorrea hirsutismo, obesidad. Algunas ademas, consultaron por infertilidad.

b) Testosterona mayor o igual a $50 \mathrm{ng} /$ $\mathrm{ml}$.

c) Relación anormal de LH/FSH y

d) Laparoscopia diagnóstica que mostrara cambios macroscópicos de androgenicidad ovárica con ovarios blancos nacarados, cápsula engrosada, quistes foliculares subcapulares y generalmente sin signos de ovulación. Adicionalmente se midió $\mathrm{PRL}$ en la mayoría de ellas.

Una vez hecho el diagnóstico se instauró tratamiento durante 3 ciclos con medroxiprogesterona $10 \mathrm{mg}$ día durante 5 días inicialmente durante el período de oligo-amenorrea y en los ciclos posteriores a partir del día 21 del ciclo. Se monitorizó ovulación con curva de temperatura basal (CTB) y se practicó seguimiento posterior basado en regularización de los ciclos, ovulación y embarazo. 
Las determinaciones hormonales se realizaron en el Laboratorio Central de Endocrinología del Hospital Universitario del Valle por técnicas de Radioinmunoensayo previamente establecidas (1).

\section{RESULTADOS}

En 16 pacientes se determinó $\mathrm{FSH}$ y LH. 28 tenían determinación de testosterona total. Determinación de PRL se realizó en 27 pacientes (Cuadro No. 1). 13 de ellas mostraron signos macroscópicos diagnósticos de S.O.A. 16 pacientes fueron llevadas a laparoscopia principalmente como parte del estudio de infertilidad (Cuadro No. 2). En todas se monitorizó sus ciclos durante y posterior al tratamiento y en 23 de las 30 pacientes se realizaron CTB confiables.

El $90 \%(n=27)$ regularizaron sus ciclos después del tratamiento cuando el seguimiento promedio fue de 5 meses. $47.8^{\circ}$, presentaron CTB ovulatorios durante algunos de los ciclos y de las que ovularon y deseaban embarazo 54\% se embarazaron, 4 a término y 2 abortos de 8 semanas de gestación. De las 16 pacientes que tienen determinación $\mathrm{LH} / \mathrm{FSH}, 11$ presentaron una relación $\mathrm{LH} / \mathrm{FSH}$ de 2.86 ó $>$ (2.86-5.86).

El $66.6 \%$ tenía valores de Testosterona mayores de $50 \mathrm{ng} / \mathrm{ml}$. No encontramos una relación absoluta entre LH/FSH $\uparrow \mathrm{y} T \uparrow$. Independientemente cada una de las determinaciones se encuentran alteradas en un porcentaje igual, es decir algunas tienen $T<50 \mathrm{ng} \circ \mathrm{LH} / \mathrm{FSH}$ $>$ 2.86. Las pacientes que mostraban $\mathrm{LH} / \mathrm{FSH}>2.68$ independiente del valor de $T$ no ovularon, aunque sí regularizaron los ciclos.

En las pacientes en que se realizó Laparoscopia y éstas eran positivas pa- ra diagnóstico de ovario androgénico $(n=16)$ y concomitantemente se determinó valores de LH/FSH, T y PRL (Cuadro No. 2) hubo mejor correlación con LH/ $\mathrm{FSH}$ alta que con niveles de $\mathrm{T}$ altos, puesto que las pacientes con Laparosco-

\section{Cuadro No. 1}

\section{PERFIL HORMONAL 30 PACIENTES CON DX.: S.O.A. Y TRATAMIENTO CON ACETATO MEDROXIPRO- GESTERONA. (Ver texto para valores)}

\begin{tabular}{rccccc}
\hline Caso No. & $\begin{array}{l}\text { LH } \\
\text { mUI/L }\end{array}$ & $\begin{array}{l}\text { FSH } \\
\text { mUI/L }\end{array}$ & LH/FSH & $\begin{array}{l}\text { PRL } \\
\text { ng/ml }\end{array}$ & $\begin{array}{l}\text { T } \\
\text { ng/nl }\end{array}$ \\
\hline 1 & 20.5 & 3.5 & 5.86 & $20-50$ & 5 \\
2 & 13.2 & 6.9 & 1.92 & $<20$ & - \\
3 & 12.1 & 5.4 & 2.24 & $<20$ & 4 \\
4 & - & - & - & $50-80$ & 3 \\
5 & - & - & - & $<20$ & 5 \\
6 & 15.2 & 4.9 & 3.09 & $20-50$ & 5 \\
7 & - & - & - & $20-50$ & 5 \\
8 & - & - & - & - & 5 \\
9 & 2.5 & 1.4 & 1.79 & $20-50$ & 5 \\
10 & - & - & - & - & 5 \\
11 & 9.4 & 3.5 & 2.68 & $<20$ & 2 \\
12 & - & - & - & $20-50$ & 3 \\
13 & - & - & - & - & 5 \\
14 & & & & $<20$ & 4 \\
15 & 10 & 3.4 & 2.94 & $<20$ & 1 \\
16 & 10.2 & 3.3 & 3.09 & $<20$ & 2 \\
17 & 16 & 5.6 & 2.86 & $<20$ & - \\
18 & 3.0 & 1.8 & 1.67 & $<20$ & 5 \\
19 & 5.3 & 3.6 & 1.47 & $20-50$ & 5 \\
20 & 20.7 & 4.0 & 5.16 & $<20$ & 3 \\
21 & & & & $<20$ & 1 \\
22 & $>50$ & 13 & 3.85 & $<20$ & 5 \\
23 & $>40$ & 11.7 & 3.42 & $<20$ & 5 \\
24 & 14 & 4.7 & 2.98 & $<20$ & 4 \\
25 & 10.5 & 3.6 & 2.92 & $20-50$ & 3 \\
26 & & & & $<20$ & 1 \\
27 & & & & $<20$ & 5 \\
28 & & & & $<20$ & 5 \\
29 & & & & $20-50$ & 5 \\
30 & & & & $<20$ & 4 \\
\hline & & & & &
\end{tabular}


Cuadro No. 2

Perfil Hormonal y Resultados en cuanto a regularización de los ciclos. BBT Bifásica y Embarazo en 16 Pacientes que se les realizó Laparoscopia Diagnóstica (Ver tex to para explicación). BBT (^) Plana; (^) ovulatoria. Hirsutismo $(\uparrow)$ positivo $\rightarrow(-)$.

\begin{tabular}{|c|c|c|c|c|c|c|c|c|}
\hline Caso No. & $\begin{array}{l}\text { Laparoscopia } \\
\text { Diagnóstica }\end{array}$ & $T$ & $\begin{array}{l}\mathrm{PRL} \\
\mathrm{ng} / \mathrm{ml} .\end{array}$ & $\begin{array}{l}\text { LH/ } \\
\text { ES }\end{array}$ & Hisrutismo & BBT & Ciclos & \\
\hline 4 & + & 3 & $50-80$ & & $\uparrow$ & $\rightarrow$ & $\mathrm{Si}$ & \\
\hline 5 & + & 5 & $<20$ & & $\uparrow$ & $\uparrow$ & $\mathrm{Si}$ & \\
\hline 7 & - & 5 & $20-50$ & & $\uparrow$ & $\rightarrow$ & $\mathrm{Si}$ & \\
\hline 8 & + & 5 & & & $\uparrow$ & $\uparrow$ & $\mathrm{Si}$ & \\
\hline 9 & + & 5 & $20-50$ & & & $\uparrow$ & $\mathrm{Si}$ & Si \\
\hline 11 & + & 2 & $<20$ & 2.68 & $\rightarrow$ & $\rightarrow$ & $\mathrm{Si}$ & \\
\hline 13 & + & 5 & & & $\uparrow$ & $\uparrow$ & $\mathrm{Si}$ & $\mathrm{Si}$ \\
\hline 15 & + & 1 & $<20$ & 2.94 & $\uparrow$ & $\rightarrow$ & $\mathrm{Si}$ & \\
\hline 16 & + & 2 & $<20$ & 3.09 & $\uparrow$ & $\rightarrow$ & $\mathrm{Si}$ & \\
\hline 20 & + & 3 & $<20$ & 5.16 & $\uparrow$ & $\rightarrow$ & $\mathrm{Si}$ & - \\
\hline 21 & + & 1 & $<20$ & & $\rightarrow$ & $\uparrow$ & $\mathrm{Si}$ & N.D. \\
\hline 22 & + & 5 & $<20$ & 3.85 & $\uparrow$ & $\rightarrow$ & $\mathrm{Si}$ & - \\
\hline 26 & + & 1 & $<20$ & & $\uparrow$ & $\rightarrow$ & Si & - \\
\hline 27 & + & 5 & $<20$ & & $\uparrow$ & $\uparrow$ & $\mathrm{Si}$ & $\mathrm{Si}$ \\
\hline 28 & - & 5 & 20 & & $\uparrow$ & $\rightarrow$ & $\mathrm{Si}$ & - \\
\hline 29 & - & 5 & $20-50$ & & $\rightarrow$ & $\uparrow$ & $\mathrm{Si}$ & Si \\
\hline
\end{tabular}

pia negativa para S.O.A., todas tenían T $>60 \mathrm{ng} / \mathrm{dl}$. Todas las pacientes con LH/ $\mathrm{FSH}$ alto eran laparoscopiapositiva. En las pacientes que no regularizaron los ciclos no se encontró ningún dato en la historia, importante de realzar. Los valores de $T$ se agruparon así:

$1=20-30 \mathrm{ng} / \mathrm{dl} ; 2=30-40 \mathrm{ng} / \mathrm{dl}$;

$3=40-50 \mathrm{ng} / \mathrm{dl} ; 4=50-60 \mathrm{ng} / \mathrm{dl}, \mathrm{y}$ $5=>60 \mathrm{ng} / \mathrm{dl}$.

\section{DISCUSION}

El uso de medroxiprogesterona como parte del estudio de pacientes con amenorrea está bien establecido. Los progestágenos tienen su indicación principal en la hemorragia uterina disfuncional anovulatoria y se han hecho varios esquemas al respecto (2). Entre los agentes progestacionales, el acetato de medroxiprogesterona, tiene la ventaja además de 
ser uno de los compuestos más potentes, el que diferente a los derivados de 19Nor-Testosterona no afecta adversamente los lípidos sanguíneos y varios parámetros de coagulación (3). El perfil hormonal en el S.O.A. incluye niveles de $\mathrm{LH}$ altos con niveles de FSH bajos (4), lo que implica mayor y más constante estimulación de la teca interna con el consiguiente aumento de producción de androstendiona (A) y su conversión periférica a estrona $(E)$. Aunque los niveles de estradiol, están reducidos en el S.O.A., éstos representan la suma de la conversión extraglandular de testosterona (5) y la interconversión de estrona a estradiol así como la secreción directa por el ovario (6). Sin embargo el hipotálamo de estas pacientes responde adecuadamente a los mecanismos de retroalimentación negativo y positivo (4-7); además cuando se usa clomífero, que es un antiestrógeno, produce liberación de LH y $\mathrm{FSH}$ comparables a los observados en mujeres normales que reciben el mismo tratamiento (8).

El hecho de que las pacientes regularan sus ciclos cuando reciben un ciclo de tratamiento con acetato de medroxiprogesterona y que, como en nuestro estudio, el $49 \%$ ovularon, implica que al menos hasta donde se ha hecho el seguimiento, hay normalización de la relación LH: FSH y la producción periférica de estrógenos secundaria a una maduración adecuada para inducir la liberación del pico de $\mathrm{LH}$ y $\mathrm{FSH}$ con la consiguiente ovulación. Esto indica que con la utilización de acetato de medroxiprogesterona se rompe el círculo vicioso que de perpetuarse lleva a los diferentes estados clínicos y a los progresivos cambios morfológicos del ovario hasta la clásica descripción clínico-patológica de Stein y Levental (9).
Figura No. 2

\section{ACCION CENTRAL DE MEDROXIPROGESTERONA EN S.O.A.}

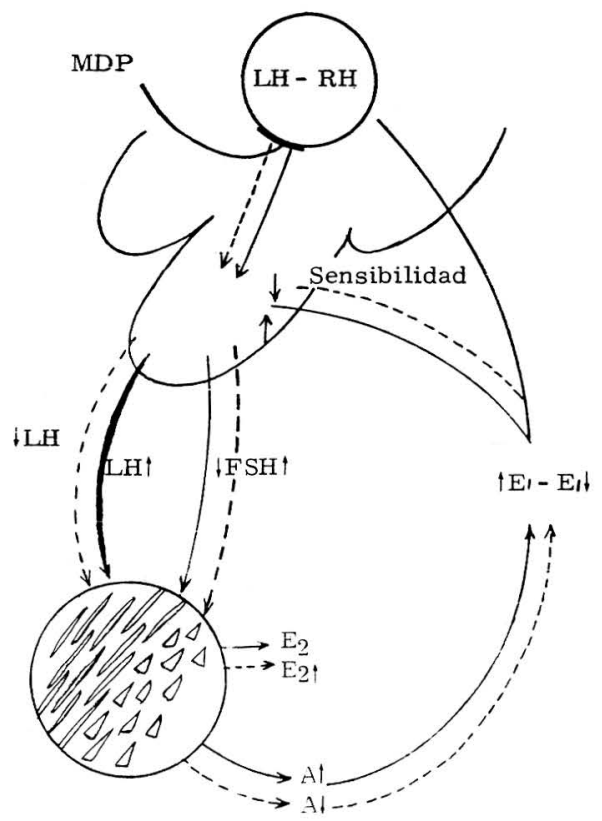

M.D.P. inhibe la secreción endógena de $L H-R H$, con consiguiente disminucion de $\mathrm{LH}_{,} \mathrm{y}$ aumento de $\mathrm{FSH}$, baja de $A$, aumerito de estrógenos $y$ disparo de mecanismos de retroalimentación positiva.

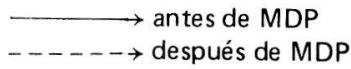

La acción antiestrogénica de la progesterona a nivel de endometrio es sobre el receptor estrogénico citoplasmático inicialmente y nuclear posteriormente, a través de un mecanismo de interferencia con la síntesis de nuevo receptor citoplasmático de estradiol (10). Además, la progesterona puede incrementar 
la inactivación de estradiol a través de la estimulación de 17 estradiol dehidrogenasa: este mecanismo disminuye la relación de estradiol a estrona en el núcleo, disminuyendo la síntesis de DNA (11) (13).

Progestágenos como acetato de medroxiprogesterona, undecilato de norgestel y acetato de noretisterona han sido administrados en forma experimental a dosis de $150 \mathrm{mg}, 100 \mathrm{mg}$ y $10 \mathrm{mg}$. día, respectivamente en mujeres post-menopáusicas normales y en todas las ocasiones hay disminución significante de $\mathrm{FSH}$ y $\mathrm{LH}$, conservando intacta !a respuesta a LH-RH Exógena, lo cual sugiere que su acción reducidora de gonadotropina es esencialmente hipotalámica inhibiendo la secreción endógena de LH-RH (12).

La acción inhibidora central de la medroxiprogesterona, especialmente de $\mathrm{LH}$, con la consecuente disminución de estimulación tecal, baja de producción androgénica y por ende estrogénica, disminuye la crónica supresión de FSH para, una vez suspendida la droga, se produzca una estimulación hipotalámica adecuada con inducción de ovulación o al menos disminución de andrógenos y producción estrogénica que no llega a niveles preovulatorios, pero que induce regularización de los ciclos, aunque persisten anovulatorios.

La acción periférica a nivel de endometrio de la medroxiprogesterona se dirige a la disminución de receptores estrogénicos. Durante el ciclo menstrual el contenido celular de receptores para estradiol es alto en la fase proliferativa (2.0 $\mathrm{pmol} / \mathrm{mg}$ de DNA), lo cual es consisten- te con la inducción por estrógenos de este receptor citoplasmático. Hacia la mitad del ciclo el número de receptores nucleares se duplica (de 0.4 a $0.9 \mathrm{pmol} / \mathrm{mg}$ de DNA) (4), lo que refleja el transporte de receptor citoplasmático al núcleo. Al tiempo de ovulación hay aproximadamente 8.000 receptores de estradiol por célula endometrial. Después de ovulación de receptores de estrógenos caen a 0.7 $\mathrm{pmol} / \mathrm{mg}$.

Los receptores citoplasmáticos disminuyen primero y luego lo hacen los receptores nucleares.

La pérdida de estos receptores para estrógenos es debido a la interferencia de la progesterona con la provisión del receptor citoplasmático para estradiol (10).

La acción antiestrogénica a nivel periférico endometrial será el otro sitio de acción de medroxiprogesterona, que implicará estimulación de los mecanismos de retroalimentación negativa, con incremento de FSH, maduración folicular, producción estrogénica adecuada y disparo de los mecanismos de retroalimenta. ción positiva con ovulación subsiguiente. Creemos que la medroxiprogesterona puede usarse con éxito en los casos de S.O.A. para regularizar los ciclos, aunque posiblemente en algunos casos, la acción sea transitoria, pero en otro porcentaje importante la duración sea más duradera o definitiva. El hecho de que en un $49^{\circ}$ o se presente ovulación, hace indispensable un método anticonceptivo diferente a los anovulatorios orales cuando no se desee embarazo, si se usa medroxiprogesterona para únicamente regularizar los ciclos. 


\section{BIBLIOGRAFIA}

1. GOMEZ, G.; ZULUAGA, A.L. Síndrome de Ovario Androgénico. Rev. Col. Obstet. Ginecol. 31: 219, 1980.

2. VORYS, N.; NERI, A.; BOUTSELIS, J. Menstrual Disfunction in Gynecologic Endocrinology. Ed. Jay. J. Gold. Harper Grow 1975, p. 213.

3. FLOWER, CH; WILBORN, W: HYDE, Barbara. Mechanisms of Uterine bleeding in post menopausal Patients receiving estrogen alone or with a progestin, Obstet. Gynecol. 61: 135, 1983.

4. YEN-SSC.; VELA, P.; RANKIN, J. Inapropiate secretion of follicle stimulating hormone and luteinizeing hormone in P.O.D. J. Clin. Endocrin. Metab. 30: 435, 1970.

5. SIITERI, P.; Mac. DONALD, P.C. Role of extrangular estrogen in human endocrinology. In Handbook of Physiology.Ed. R.O. Green and E. Astwood. pag. 615, 1973.

6. LONGCOPEC. WILLIAMS, K. The metabolism of estrogens in normal women after pulse injection of $\mathrm{H}^{3}$ estradiol and $\mathrm{H}^{3}$ estrone. J. Clin. Endocrin. Metab. 38: $602,1974$.

7. REBAR, R.; JUDD, H.L.; YEN, SC.; RAKKOF， J.; VAN DEN BERG, G.; NAFTOLIN, F. Characterization of the inappropiate gonadotropin secretion in P.O.C. J. Clin. Invest. 57: 1320, 1976.

8. YEN-SCC .; Ve, P.; RYAN, K. Effect of clomiphene cetrate in P.O.S.: Relations- hip between serum gonadotropin and corpus luteun function. J. Clin. Endocrin. Metab. 31: 7, 1970.

9. STEIN, I.F.; LEVENTHAL, M.L. Amenorrhea associated with bilateral polycystic ovaries. Am. J. Obstet. Gynecol. 29: $181,1935$.

10. HSUEH, A.W. PECK E., CLARK J. Progesterone antagonism of the estrogen receptor and estrogen induced uterine growth. Nature (lond) 254: 337, 1975.

11. WHITEHEAD, M. TOWSEND B., PRYSE DAVIES, RYDER. T., KING, J.R. Effects of estrogens and progesting on biochemistry and morphology of the post menopausal endometrium. N. Engl. J. Med. 205, 1599, 1981.

12. FRONCHIMONT, P. GASPARD, J.R., VAN CAUWENBERGE, DEPAS, E. Some Aspects of hypothalamus-hypophysealovarian feedback mechanism in women "The Endocrine Function of the Ovary". p. 119. Ed. Jones, Serio, Eusti. Academic press, 1976.

13. TSENG, L. GURPIDE, E. Estradiol and 20 -dehydroprogesterone dehidrogenase activities in human endometrium during the menstrual cycle. Endocrinology 94: $419,1974$.

14. BAYARD, F., DAMILANO, S., ROBEL, P. and BAULIEU, E. Cytoplasmatic and progesterone receptors in human endometrium. J. Endocrinol. Metab. 46: 635, 1978. 